\title{
USO DE LA MASTOFAUNA SILVESTRE EN LA COMUNIDAD CAFETALERA DE CUMBRES DE HUICICILA, COMPOSTELA, NAYARIT, MÉXICO
}

\section{USE OF WILD MASTOFAUNA IN THE COFFEE PRODUCING COMMUNITY CUMBRES DE HUICICILA, COMPOSTELA, NAYARIT, MEXICO}

\author{
Fátima Carolina Medina-Gutiérrez I I Juan Pablo Ramírez-Silva ${ }^{1}$
}

\begin{abstract}
${ }^{1}$ Maestría en Ciencias para el Desarrollo, Sustentabilidad y Turismo. Unidad Académica de Turismo. Universidad Autónoma de Nayarit. Ciudad de la Cultura “Amado Nervo". Tepic, Nayarit. México. CP 63155.
\end{abstract}

\section{Resumen}

El objetivo del presente trabajo fue analizar la percepción y usos de los mamíferos silvestres en un área cafetalera de la comunidad Cumbres de Huicicila, municipio de Compostela, Nayarit. Se utilizaron técnicas etnozoológicas tales como aplicación de encuesta sobre el usos y costumbres que tienen en relación con los mamíferos. Asimismo, se usaron cámaras-trampa con un esfuerzo de muestreo de 1,260 días/trampas. En total se obtuvieron 531 videos y 373 registros válidos de mamíferos silvestres de 10 especies pertenecientes a cinco órdenes y siete familias, tres de ellas enlistadas en la NOM-059-SEMARNAT-2010 y la lista roja de la UICN (Panthera onca, Leopardus pardalis y Herpailurus yagouaroundi). A través de las herramientas etnozoológicas se identificaron 15 especies de mamíferos y cinco órdenes. Las personas de la comunidad afirmaron darle algún tipo de uso a los mamíferos que conocen, entre los que destacan el alimenticio, medicinal, tráfico ilegal (venta de especies), cacería e inclusive se mencionaron algunos conflictos de relación depredador-humano. A pesar de lo mencionado, la participación de la comunidad fue positiva. Trabajos de este tipo pueden favorecer la sensibilización hacia la vida silvestre y así, la ejecución de pro-

Revisado: 19 de noviembre de 2019; aceptado: 16 de diciembre de 2019; publicado: 30 de diciembre de 2019. Autor de correspondencia: Juan Pablo Ramírez Silva, pablor@uan.edu.mx

Cita: Medina-Gutiérrez, F.C. y J.P. Ramírez-Silva. 2019. Uso de la mastofauna silvestre en la comunidad cafetalera de Cumbres de Huicicila, Compostela, Nayarit, México. Revista Mexicana de Mastozoología, nueva época, 9(2):29-42. ISSN: 2007-4484.www. revmexmastozoologia.unam.mx

\section{ReleVAnCia}

El presente estudio es un ejemplo de cómo los trabajos de uso de mastofauna, aportan valiosos conocimientos tradicionales que pueden influir en los pobladores que deseen colaborar en programas de conservación para las especies silvestres nativas.

gramas de manejo, uso y conservación de mamíferos podría tener éxito y a su vez ayudar a la subsistencia de la comunidad.

Palabras clave: Cafetales, etnomastozoología, jaguar, mesomamíferos, uso de fauna silvestre.

\section{Abstract}

In this paper we analyzed the perception and uses of the wild mammals by the population that lives in Cumbres de Huicicila community, municipality of Compostela, Nayarit. The information was complemented by the use of camera traps, from which we obtained 531 videos with 373 valid records of wild mammals, with 1,260 days/traps sampling effort. We recorded 10 species belonging to five orders and seven families, three of such (Panthera onca, Leopardus pardalis and Herpailurus yagouaroundi) are listed in NOM-059-2010 and the IUCN red list. The habitants of Cumbres de Huicicila community identify 20 species of mammals, including the 10 species registered by the camera traps, the people of the community know the mammals and are used, they mentioned as food, medicine, illegal trafficking, hunting for leisure and ornaments and even some predatory-human relationship conflicts. Despite them, 
community participation was positive, we believe that this works can contribute to create the awareness of wildlife and thus, the implementation of mammalian management, use and conservation programs could be successful and help the community to increase quality live.

Key words: Coffee plantations, ethnozoology, jaguar, medium-sized mammals, use of wildlife.

\section{INTRODUCCIÓN}

Gracias a su historia evolutiva, posición geográfica y diversidad de paisajes y climas, México es un país megadiverso (CONABIO, 1998), que, a pesar de alojar una gran cantidad de especies en su territorio, para conocerlas es necesario seguir estudiándolas con mayor detalle. En México se tienen registrados 13 órdenes, 46 familias, 202 géneros y 545 especies de mamíferos, de las cuales 169 son endémicas del país (Ceballos y Arroyo-Cabrales, 2012). En el estado de Nayarit estudios recientes han documentado que cuenta con 141 especies de mamíferos terrestres, con lo que se posiciona entre los cinco estados con mayor diversidad de mamíferos del país (Hernández-Cadena, 2015; Ramírez-Silva et al., 2016).

En la actualidad, las principales amenazas que enfrentan los mamíferos de Nayarit son: la pérdida y fragmentación de hábitat, relacionado con el cambio de uso de suelo con fines agrícolas y ganaderos; el acelerado crecimiento de la industria hotelera, principalmente en la zona costera del sur del estado; y la cacería, que sigue siendo una práctica común es las zonas rurales. Los mamíferos son las especies más afectadas por esta práctica (Ramírez-Silva et al., 2016).

El grupo taxonómico de los mamíferos es bastante conocido por muchas comunidades indígenas y rurales (Medina-Torres et al., 2015). Por siglos han desempeñado un papel trascendental en el desarrollo social y económico en diversas poblaciones humanas. A su vez, son un importante componente de la biodiversidad. Representan valores éticos, culturales, económicos, políticos, ecológicos, recreacionales, educativos y científicos, que han ido de la mano con el desarrollo de la humanidad y la historia de la tierra (Córtes-Gregorio et al., 2013). Esto se ha reflejado en su uso como fuente de alimento, medicina, vestido, e incluso han sido empleados como fuerza de trabajo y compañía. Su relación es tan estrecha que han llegado a ocupar un lugar privilegiado dentro de las creencias de muchas culturas alrededor del mundo, por lo que es innegable que la subsistencia de la especie humana se debe a los conocimientos de nuestros ancestros en torno a la vida silvestre (Contreras-Díaz y Pérez-Lustre, 2008).

Las comunidades rurales desempeñan un importante papel en el buen funcionamiento de las estrategias de conservación, pues son ellas quienes tienen un dominio pleno sobre el territorio y poseen conocimientos en torno al uso y manejo de los recursos naturales que les rodean (Bocco et al., 2000). Esta situación es especialmente importante en México, porque las zonas que poseen los menores índices de desarrollo humano y más pobreza albergan la mayor parte de la biodiversidad. Por ejemplo, el $80 \%$ de los recursos forestales se ubican en territorio donde habitan comunidades indígenas y ejidos campesinos, por lo que es importante conocer la manera en que usan y perciben la naturaleza, pues estas prácticas se convierten en sus principales factores de subsistencia (Bocco et al., 2000; Rosas-Rosas et al., 2015).

El estudio del uso que le dan las comunidades a la mastofauna es un elemento indispensable para comprender la percepción que tienen sobre las especies, robustecer el conocimiento y con ello sentar la base para el diseño de programas de manejo y conservación que atiendan los intereses y necesidades del contexto social. En Nayarit no se han realizado investigaciones que relacionen a los mamíferos silvestres con aspectos etnobiológicos, pero se han documentado en otros estados. Cómo el estudio de Medina-Torres et al. (2016) que compararon una comunidad indígena (Yoreme) con una comunidad mestiza (Yori) al norte de Sonora. Por medio de entrevistas con cazadores determinaron los principales usos de la fauna silvestre en las comunidad: alimentación, uso artesanal, medicinal y ritual. El uso no varió entre comunidades, pero sí las especies. Dicho estudio brindó información valiosa para la gestión del aprovechamiento cultural y de subsistencia de las comunidades indígenas de Sinaloa.

Por otra parte, en la Sierra occidental del estado de Aguascalientes se registró el uso de los mamíferos mejor conocidos por las personas de la tercera edad y los cazadores. Destacaron especies de valor cinegético, medicinal, así como las llamadas especies problemáticas; este estudio contribuyó al rescate del conocimiento de los usos tradicionales 
que se le da a la fauna local, pues los autores consideran que son conocimientos que se han ido perdiendo con las nuevas generaciones (Alcalá y De la Riva-Hernández, 2016).

El objetivo de este trabajo fue analizar la percepción y los usos que se le da a los mamíferos silvestres en la comunidad cafetalera de Cumbres de Huicicila, Compostela, Nayarit; donde la principal actividad económica es el corte y agroindustria de café de pequeños productores bajo un sistema rústico de montaña.

\section{Metodología}

\section{Área de estudio}

La comunidad de Cumbres de Huicicila es una comunidad ejidal de 40,918.55 ha (PHINA, 2017) que cuenta con una población de 467 habitantes (INEGI, 2010) y se ubica a 20 km de la cabecera municipal dentro de Compostela, al sureste del estado de Nayarit, México, entre los $21^{\circ} 26^{\prime} 67^{\prime \prime}$ de latitud y $-105^{\circ} 014^{\prime} 444^{\prime \prime}$ de longitud, con una altitud que va de los 919 a 1,060 msnm. Forma parte de la provincia fisiográfica de la Faja Volcánica Transmexicana, donde se pueden encontrar distintos tipos de vegetación como selva baja subcaducifolia secundaria, selva perennifolia, bosques templados (donde predominan bosques de encino) y un bosque mesófilo de montaña que rodea a la comunidad de Cumbres de Huicicila, como también, pequeñas áreas de sembradíos cafetaleros. La localidad cuenta con relieves importantes como el cerro "El Molote", el cual se encuentra en las coordenadas $21^{\circ} 16^{\prime} 54.37^{\prime \prime} \mathrm{N}$ de latitud y $105^{\circ} 0$ '47.88"O de longitud con una altura de $1,035 \mathrm{msnm}$. Este cerro se ubica dentro del territorio del ejido a una distancia de $4.1 \mathrm{~km}$ de la comunidad (Figura 1).

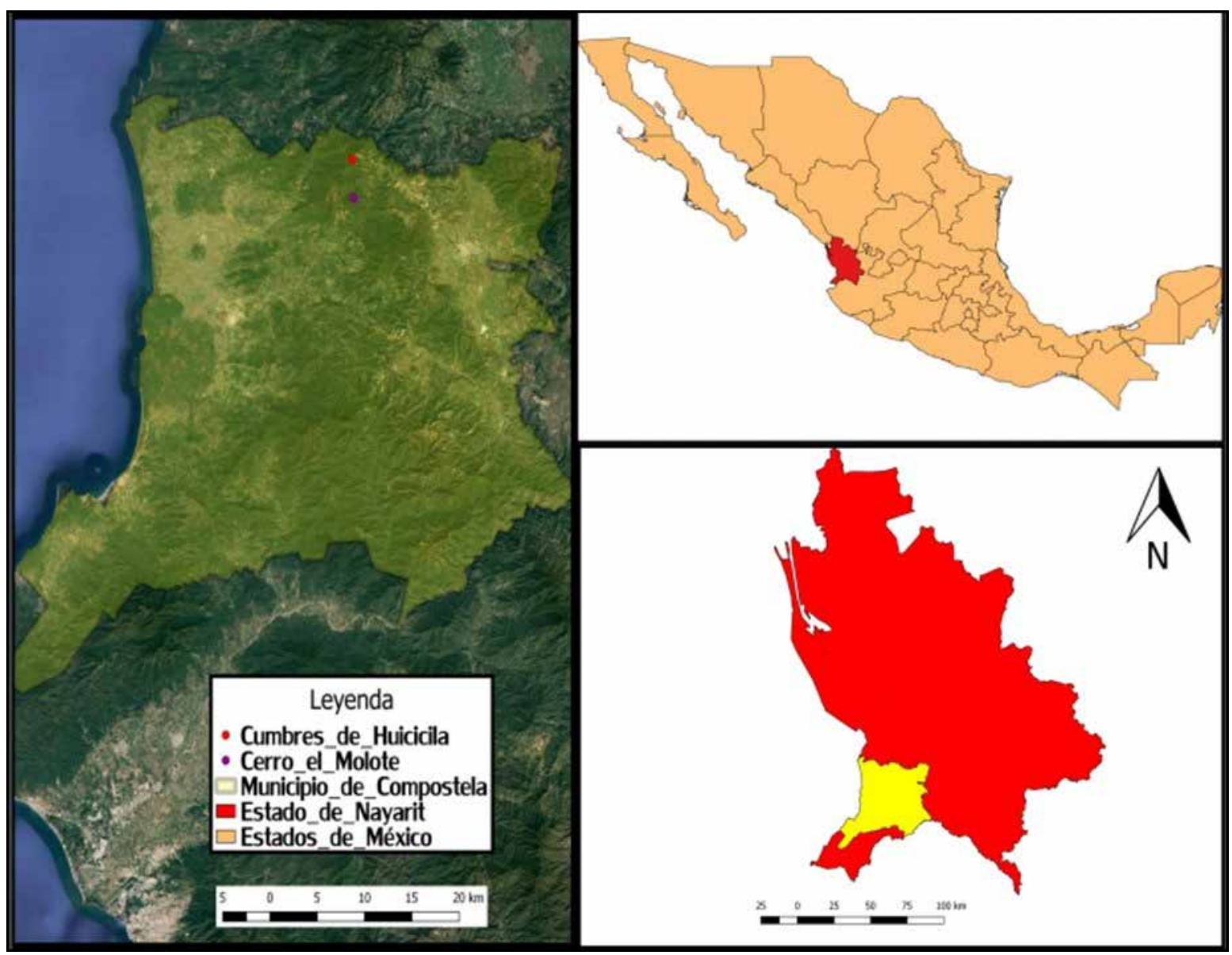

Figura 1. Localización de la Comunidad Cumbres de Huicicila (Punto rojo) y Cerro El Molote (Punto morado) en el municipio de Compostela (amarillo), Nayarit (rojo). 


\section{Inventario de mastofauna}

Para esta investigación se realizaron recorridos en campo, con el apoyo de las autoridades ejidales y las personas de la comunidad interesadas en colaborar. Los recorridos se realizaron en el cerro El Molote para identificar los sitios posiblemente más transitados por la mastofauna, corroborados por el registro de rastros y huellas de mamíferos.

También se realizó video trampeo, para lo cual se colocaron cinco cámaras-trampa (marca Cuddeback, modelo 1279), separadas por al menos 10 $2 \mathrm{~km}$ de distancia. De acuerdo con Chávez et al. (2013), se procuró poner las cámaras en lugares con senderos, brechas o sitios de alimentación natural sin atrayentes. Cada estación de muestreo fue geo-referenciada y las cámaras-trampa permanecieron activas de mayo del 2016 a enero del 2017. Las cámaras fueron revisadas cada mes aproximadamente y fueron programadas para hacer una toma de video con 20 segundos de duración, con un retraso de cuatro minutos.

Los videos se revisaron y se clasificaron como "registros válidos", para reducir la posibilidad de repetir múltiples videos de un mismo individuo que no pudiera aportar nada nuevo en un lapso de tiempo determinado, para lo que se usaron los siguientes criterios: 1) se consideró un registro válido, si se documenta en video la presencia de una especie capturada en un mismo sitio y dentro de un periodo no mayor a 60 minutos; 2) si se registra la presencia de una especie en video y es interrumpido por el registro de otra especie ( $\sin$ importar que hayan transcurrido menos de $60 \mathrm{~min}$ ), se consideran como registros válidos distintos; y 3) si en el mismo video aparecen más de dos individuos se consideran registros independientes (Godínez, 2014).

Posteriormente se calculó el porcentaje de videos efectivos por medio de la fórmula propuesta por Lozano-Rodríguez (2010):

\section{Porcentaje de videos efectivos= registros váli- dos /videos totales}

Registros válidos= se refieren al número de videos que representan alguna especie de mamífero silvestre como registro único en un lapso de tiempo.

Videos totales $=$ número de videos captados, incluyendo aquellos donde el sensor se activa por diver- sas causas (movimiento de maleza, hojas, insectos, presencia de humanos u otro tipo de fauna).

Para evaluar el esfuerzo de muestreo empleado y evaluar la fiabilidad se analizaron las curvas de acumulación de especies con el programa de EstimateS versión 9.1. (Colwell, 2013), por medio de los estimadores no paramétricos Chao 1 y ACE. A partir de los videos se elaboró un listado de especies de mamíferos. Las especies fueron identificadas con ayuda del libro de mamíferos silvestres de México (Ceballos y Oliva, 2005) y la guía de campo de anfibios, reptiles, aves y mamíferos del occidente de México (Myska, 2015). Para la nomenclatura se siguió a Ramírez-Pulido et al., (2014).

Para determinar la confiabilidad del inventario realizado se efectuaron curvas de acumulación de especies, por medio de los estimadores no paramétricos Chao 1 y ACE. El estimador Chao 1 determina el número de especies en una comunidad basado en el número de especies raras (Moreno, 2001) y el estimador ACE se utiliza para las estimaciones de 10 o menos individuos por muestra (Bautista-Hernández et al., 2013).

El valor de abundancia de cada una de las especies registradas se obtuvo con la siguiente fórmula:

Abundancia $=$ Registros sp/Registros spp $\times 100$, en donde:

Registros sp: número de registros válidos de la especie $\mathrm{x}$.

Registros spp: número total de registros obtenidos para todas las especies.

Por último, se identificaron aquellas especies incluidas bajo alguna categoría de protección en la Norma Oficial Mexicana (NOM-059-SEMARNAT-2010) y en la lista de la Unión Internacional para la Conservación de la Naturaleza (IUCN).

Percepción sobre el uso, la diversidad y conservación de la mastofauna

Para este estudio se aplicó una encuesta a 59 personas de la comunidad, con respuestas cerradas de opción múltiple. Se cuidó el uso de un vocabulario sencillo, respetuoso y se usaron los nombres comunes. Todos los encuestados hablaban español, sin embargo, en algunos casos se utilizaron imágenes 
de las especies para evitar confusiones. La encuesta se realizó para conocer la percepción que los habitantes tienen con respecto a la conservación y a las posibilidades de manejo de la biodiversidad, así como el conocimiento sobre la mastofauna de la región. Se emplearon preguntas tales como ¿Cuáles son las especies de mamíferos que habitan en esta zona? ¿Qué uso se les da? ¿Te gustaría que existiera un programa de conservación en tu comunidad?, entre otras. La encuesta fue realizada al azar a personas mayores de edad. Para estimar el número de cuestionarios que serían necesarios aplicar, se utilizó la siguiente fórmula (Carmona, 2015).

En donde:

$$
n=\frac{\left(N \sigma^{2} Z^{2}\right)}{(N-1) e^{2}+\sigma^{2} Z^{2}}
$$

n: el tamaño de la muestra

N: tamaño de la población

б: desviación estándar

Z: valor obtenido mediante niveles de confianza

e: límite aceptable del error de muestra

Asímismo, para registrar la información referente a las problemáticas de conservación de la fauna local se realizaron entrevistas a profundidad a los actores clave de la comunidad (Comisariado ejidal del Ejido Cumbre de Huicicila, técnico forestal del ejido, dueño del predio donde se trabajó y un productor local).

\section{Resultados}

\section{Inventario de mastofauna}

Con un esfuerzo de muestreo de 1,260 días trampas, se obtuvieron 531 videos, de ellos 373 fueron considerados "registros válidos" correspondientes al $70.24 \%$ de los videos. De ellos, la mayoría (76\%) corresponden a registros de mamíferos silvestres, mientras que el $15 \%$ pertenece a registros de humanos y el $9 \%$ a registros de aves (Figura 2). En esta área, aunque es poco transitada, se realizan trabajos con los cultivos de café, como corte de plantas, siembra de café y mantenimiento de caminos. Cabe señalar que durante el tiempo que estuvieron colo-

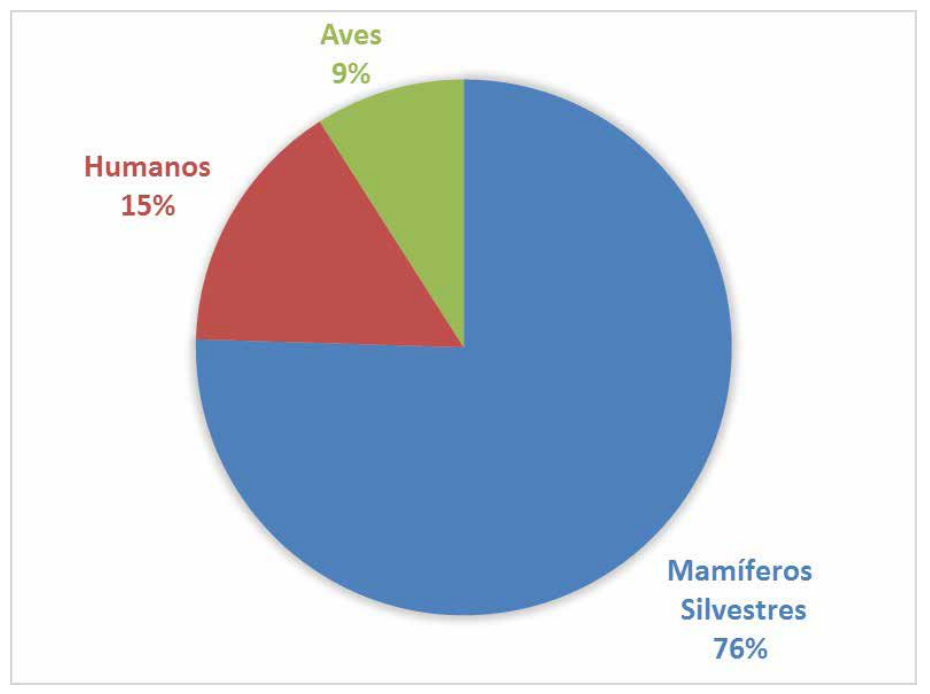

Figura 2. Porcentaje de registros válidos del muestreo con cámaras trampa de mamíferos en Cumbre de Hucicila, Nayarit.

cadas las trampas se detectó la presencia de algunos cazadores en la zona, ajenos a la comunidad.

\section{Lista de especies}

Se registraron 10 especies de mamíferos pertenecientes a cinco órdenes y siete familias; las especies registradas fueron el jaguar (Panthera onca), puma (Puma concolor), coatí o tejón (Nasua narica), venado cola blanca (Odocoileus virginianus), tlacuache (Didelphis virginiana), pecarí de collar (Dicotyles angulatus), armadillo de nueve bandas (Dasypus novemcintus), jaguarundi (Herpailurus yagouaroundi), ocelote (Leopardus pardalis) y ardillón (Otospermophilus variegatus). De las especies registradas, tres se encuentran consideradas en la NOM-059-SEMARNAT-2010 bajo la categoría en peligro de extinción ( $P$. onca y $L$. pardalis) y amenazada ( $H$. yagouaroundi; SEMARNAT, 2010), además el jaguar está considerado por la IUCN en la categoría de casi amenazado (Figura 3).

\section{Curva de acumulación}

En la gráfica de curva de acumulación de especies (Figura 4) puede observarse que las curvas tienden a estabilizarse después del tercer mes de muestreo y que los valores esperados por Chao 1 coinciden con los valores observados, mientras que, de acuerdo con el estimador ACE, se estima que se documentaron $96.80 \%$ de la diversidad esperada. Con base en lo anterior, se puede decir que el es- 


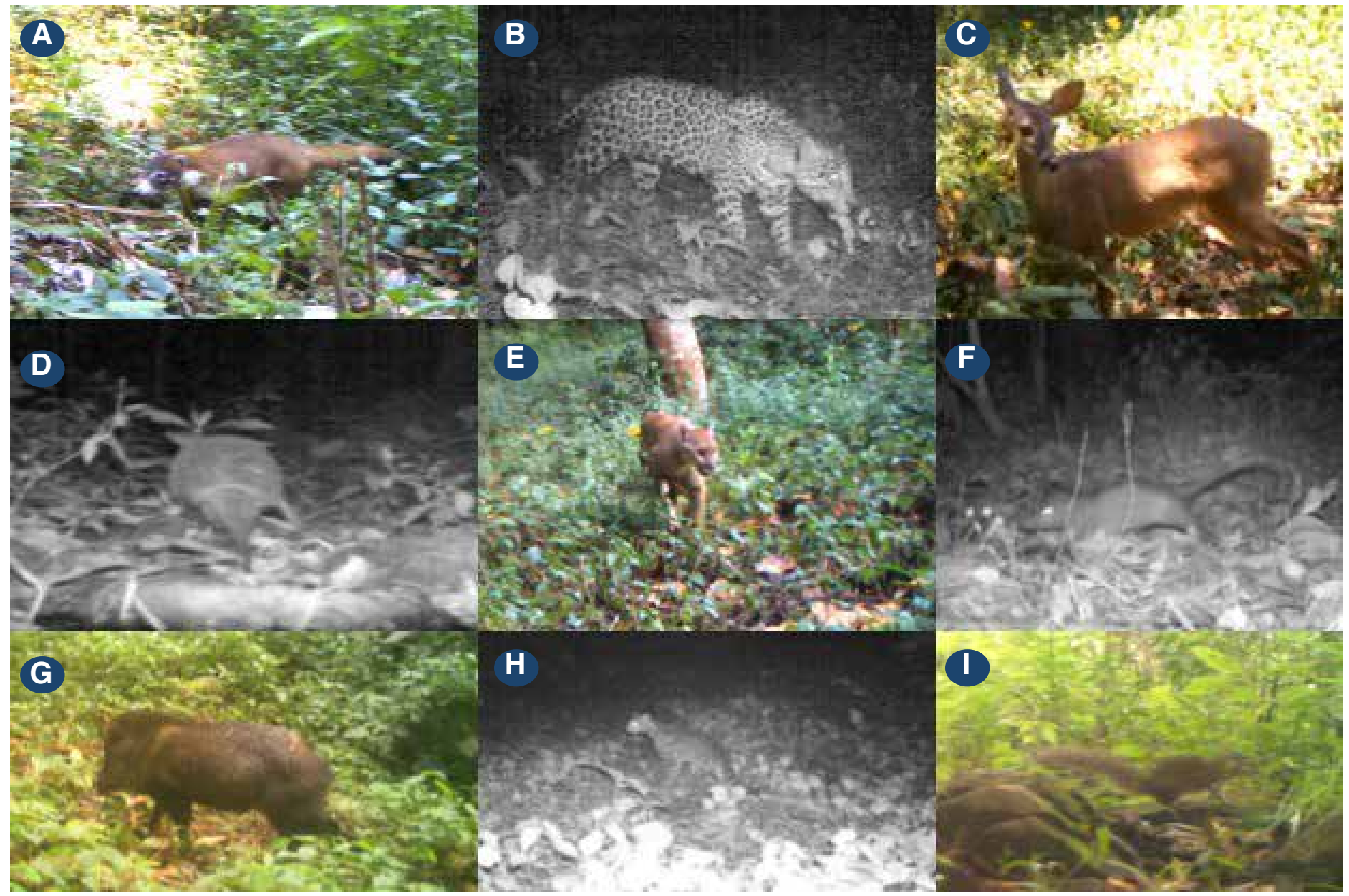

Figura 3. Mamíferos silvestres captados por las cámaras trampa en Cumbre de Huicicila, Nayarit. A) Coatí (Nasua narica), B) Jaguar (Panthera onca), C) Venado cola blanca (Odocoileus virginianus), D) Armadillo nueve bandas (Dasipus novemcintus), E) Puma (Puma concolor), F) Tlacuache común (Didelphis virginiana), G) Pecari de collar (Dicotyles angulatus), H) Ocelote (Leopardus pardalis) e I)Ardillón (Otosphermofilus variegatus).

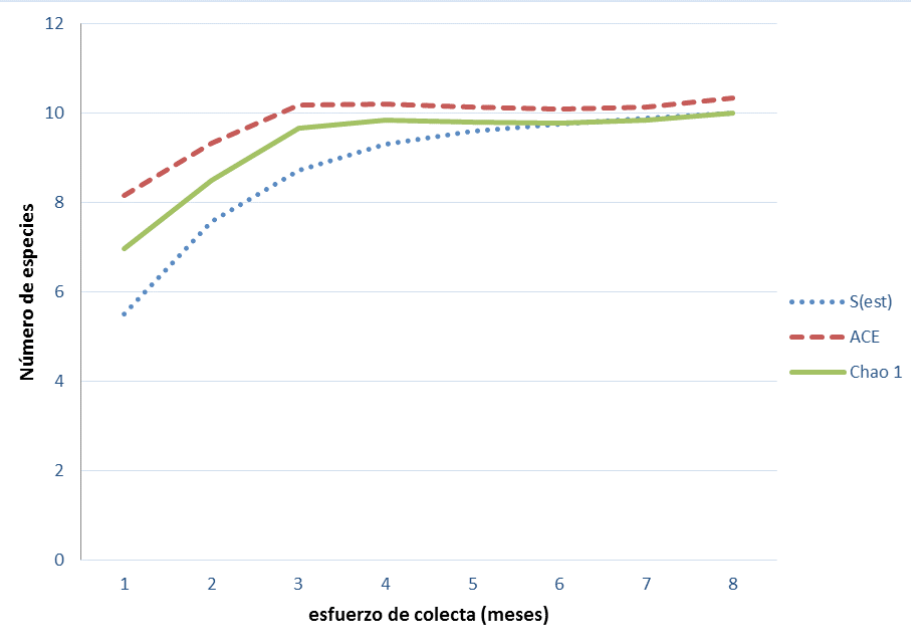

Figura 4. Curvas de acumulación de especies de mamíferos mediante el muestreo de cámaras trampa en el cerro El Molote, Cumbre de Huicicila, Compostela, Nayarit. 
fuerzo de muestro fue satisfactorio y los resultados confiables.

\section{Abundancia}

Mediante el método de video-trampeo se pudo registrar que el coatí ( $N$. narica) fue la especie que mostró la mayor abundancia relativa con un porcentaje de registros del $55.12 \%$, seguida por el venado cola blanca (O. virginianus) con un $13.24 \%$ y pecarí de collar (D. angulatus) con $6.41 \%$. El jaguarundi $(H$. yagouaround $i)$ obtuvo el porcentaje más bajo de abundancia relativa al contar con un solo registro y un porcentaje de $0.42 \%$, seguido del ocelote $(L$. pardalis), el jaguar ( $P$. onca) y el tlacuache común ( $D$. virginiana); estos últimos con $3 \%$ de abundancia.

\section{Percepción sobre el uso, la diversidad y con- servación de la mastofauna}

La edad de los encuestados fluctuó entre los 23 y 77 años, y la mayoría llevan viviendo toda su vida dentro de la comunidad. El $73 \%$ son hombres y el $26 \%$ mujeres, cuyo nivel de estudio va desde la primaria, en su mayoría concluyendo sólo el segundo o cuarto año (33\%), secundaria (33\%), preparatoria $(14 \%)$, técnico universitario $(3 \%)$ y universidad (15\%); sólo el $2 \%$ no contó con algún nivel de escolaridad. En cuanto a la ocupación, las principales actividades laborales son caficultor (53\%), ama de casa $(20 \%)$, estudiantes $(10 \%)$, comerciantes $(9 \%)$ empleados de gobierno (5\%) y jornaleros (3\%).

En la encuesta realizada se identificó la presencia de 15 especies de mamíferos, tres a nivel de familia y dos a nivel de género, que habitan dentro del territorio de la comunidad (10 de ellas se registraron en los videos de las cámaras-trampa). Las especies fueron: venado cola blanca ( $O$. virginianus), coatí ( $N$. narica), jabalí ( $D$. angulatus), armadillo ( $D$. novemcintus), ardilla (O. variegatus), jaguar ( $P$. onca), tlacuache $(D$. virginiana), mapache (Procyon lotor) los cuales no se registraron en el video-trampeo, el puma ( $P$. concolor), ocelote (Leopardus pardalis), jaguarundi $(H$. yagouarundi), coyote (Canis latrans), zorra gris (Urocyon cinereoargenteus), tlacuachin (Tlacuatzin canescens), lince (Lynx rufus). Cinco mamíferos se identificaron a nivel de familias o género: conejo (Sylvilagus sp.), comadreja (Mustelidae), tuza (Geomyidae), ratones silvestres (Rodentia) y zorrillo (Mephitidae), los cuales no se registraron en las cámaras-trampa o no fue posible su identifica- ción. Al preguntar sobre las especies avistadas la respuesta fue bastante similar a la de las especies más conocidas, pues el venado, el coatí y el jabalí fueron las que encabezaron la lista (Cuadro 1).

En relación con aspectos de conservación y cuidado de las especies, el $93 \%$ de las personas encuestadas consideran importante la conservación de los mamíferos silvestres dentro del ejido, un $5 \%$ no sabe si es importante y un $2 \%$ dice que no es importante. Los que dieron la respuesta positiva manifestaron que dicha importancia es relevante para mantener el equilibrio de las cadenas tróficas (22\%), porque los mamíferos son parte de la naturaleza (16\%), para que puedan ser conocidas por las nuevas generaciones $(10 \%)$, por sus usos medicinales y alimenticios (10\%), para que se pueda conservar todo el hábitat (10\%), para que aumenten las poblaciones de animales $(6 \%)$ porque si no se conservan se pueden extinguir $(5 \%)$, por estética de los cerros $(3 \%)$, porque están en peligro $(2 \%)$ y por el simple gusto de cuidarlos (2\%). En contraparte, las personas que dieron la respuesta "no sé si sea importante" o "no es importante", mencionaron que las razones son que puede ser peligroso conservar a ciertas especies, haciendo referencia específicamente al jaguar, el puma y el coatí por ser un animal dañino, de igual manera, estos felinos perjudican a los animales que ellos consideran buenos como el venado o el jabalí.

Con base en la pregunta anterior, también se les preguntó si consideran más importante un mamífero que otro; el $81 \%$ de los encuestados mencionó que la conservación debe ser igual para todas las especies, un $14 \%$ dijo que considera que se debe conservar más al venado, siguiéndole el jabalí y el jaguar con un $2 \%$ y finalmente el $1 \%$ mencionó al coatí como una especie especialmente importante que conservar.

Referente al uso de la mastofauna silvestre, la especie más mencionada fue el venado como alimento y como un animal para cacería deportiva $(26 \%)$, siguiéndole el coatí como alimento y también como un remedio tradicional para los cólicos (25\%), el armadillo como alimento, como animal de ornato y como remedio casero para la bronquitis (la grasa del caparazón; $21 \%$ ), el jabalí como alimento $(8 \%)$, la ardilla como alimento $(6 \%)$, el mapache como alimento (3\%), el tlacuache como alimento y remedio casero para granos, salpullido y ampollas (2\%) y el zorrillo como remedio casero para enfermedades respiratorias en general (su glándula odo- 
Cuadro 1. Lista de especies de mamíferos registradas por medio de las encuestas realizadas en la comunidad de la Cumbre de Huicicila, Compostela, Nayarit. Se indica porcentaje de menciones y si se obtuvo su registro con las cámaras. (ND= especie no determinada).

\begin{tabular}{|c|c|c|c|c|c|}
\hline Orden & Familia & Especie & Nombre común & Encuesta & $\begin{array}{l}\text { Presencia } \\
\text { en cámaras }\end{array}$ \\
\hline \multirow[t]{2}{*}{ Didelphimorphia } & \multirow[t]{2}{*}{ Didelphidae } & Didelphis virginiana & Tlacuache & $7 \%$ & $\mathrm{Si}$ \\
\hline & & Tlacuatzin canenses & Tlacuachín & $>1 \%$ & No \\
\hline Cingulata & Dasypodidae & Dasypus novemcintus & Armadillo & $11 \%$ & $\mathrm{Si}$ \\
\hline \multirow{10}{*}{ Carnivora } & Procyonidae & Procyon lotor & Mapache & $6 \%$ & No \\
\hline & \multirow{5}{*}{ Felidae } & Panthera onca & Jaguar & $9 \%$ & Si \\
\hline & & Puma concolor & Puma & $5 \%$ & Si \\
\hline & & Leopardus pardalis & Ocelote & $5 \%$ & $\mathrm{Si}$ \\
\hline & & $\begin{array}{l}\text { Herpailurus } \\
\text { yagouaroundi }\end{array}$ & Jaguarondi & $4 \%$ & Si \\
\hline & & Lynx rufus & Lince & $1 \%$ & No \\
\hline & \multirow[b]{2}{*}{ Canidae } & Canis latrans & Coyote & $2 \%$ & No \\
\hline & & $\begin{array}{c}\text { Urocyon } \\
\text { cinereoargenteus }\end{array}$ & Zorro & $1 \%$ & No \\
\hline & Mephitidae & ND & Zorrillo & $2 \%$ & No \\
\hline & Mustelidae & Mustela sp. & Comadreja & $1 \%$ & No \\
\hline \multirow{3}{*}{ Rodentia } & Sciuridae & $\begin{array}{c}\text { Otospermophilus } \\
\text { variegatus }\end{array}$ & Ardilla & $10 \%$ & Si \\
\hline & Geomyidae & Thomomys sp. & Tuza & $>1 \%$ & No \\
\hline & Cricetidae & ND & Ratón & $>1 \%$ & No \\
\hline Lagomorpha & Leporidae & Sylvilagus sp & Conejo & $1 \%$ & No \\
\hline \multirow[t]{2}{*}{ Artiodactyla } & Cervidae & Odocoileus virginianus & Venado & $14 \%$ & $\mathrm{Si}$ \\
\hline & Tayassuidae & Dicotyles angulatus & Pecarí/jabalí & $11 \%$ & $\mathrm{Si}$ \\
\hline
\end{tabular}

rífera; $1 \%$; el 13\% mencionó que no tienen uso alguno (Figura 5).

La cacería ilegal fue detectada como la mayor amenazada para la biodiversidad dentro de la comunidad con un $72 \%$ de las respuestas. Asimismo, se comentó que el desmonte de bosques y la aplicación de herbicidas pueden ser nocivos para los animales con un $5 \%$ de respuestas cada una; también se identificó como amenaza a los perros ferales (3\%), al igual que la urbanización y la extracción de especies con un $1 \%$ (5.9 personas). Sin embargo, un $8 \%$ dijo que no había peligro alguno para las especies y otro $5 \%$ identificó al jaguar como un grave peligro para las especies buenas que deambulan por la región.
Es importante señalar que gracias a comentarios de personas de la misma comunidad, fue posible determinar que quienes clasificaron al jaguar como peligro para la biodiversidad o que no existe ninguna amenaza en la localidad, son gente que se dedica a la cacería. Esto tiene lógica ya que más de la mitad de los encuestados clasificó la cacería ilegal como el mayor problema para la biodiversidad, el cual se ve favorecido por los usos y costumbres de los pobladores que hacen uso de la fauna silvestre, aunado a la cacería deportiva.

El $54 \%$ de la población tiene conocimiento de que existe algún programa de conservación dentro de la comunidad, de los cuales el más popular es el programa de pagos por servicios ambienta- 


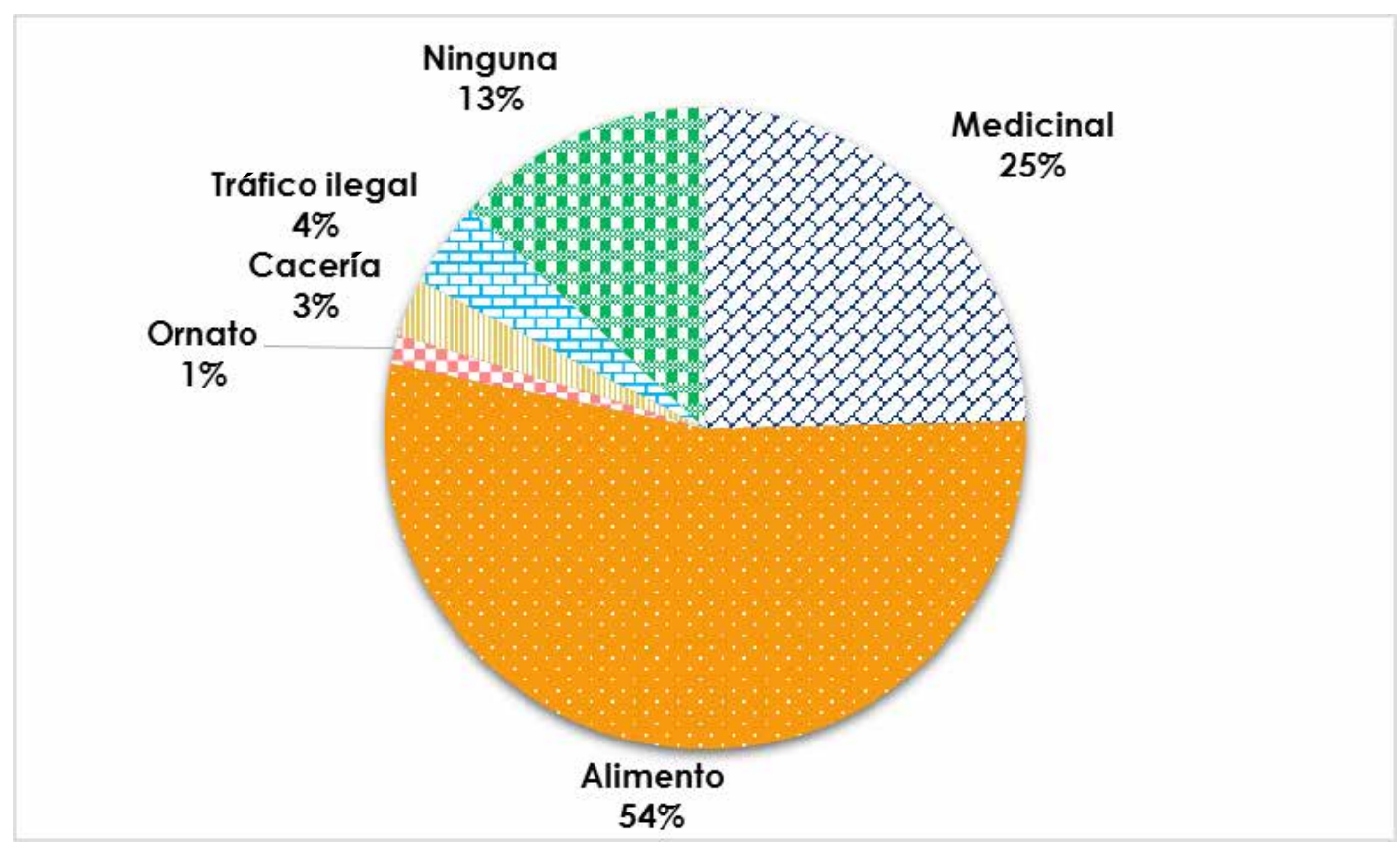

Figura 5. Porcentaje de los tipos de usos que le da la comunidad a los mamíferos en Cumbre de Huicicila, Nayarit.

les, aunque consideran que el beneficio es para un particular y a los vigilantes ambientales; asimismo consideran que estos últimos no reportan ninguna anomalía ambiental a las autoridades.

Con base en lo anterior, a la mayoría de los encuestados les gustaría que existiera un programa de conservación que permitiera que se viera beneficiada toda la comunidad. La principal opción es el pago por servicios ambientales (46\%) como el programa más solicitado por los pobladores, seguido del ecoturismo (12\%) como otra opción de conservación, una Unidad de Manejo para la Conservación de la Vida Silvestre (UMA) específicamente de O. virginianus $(10 \%)$ y la creación de un Área Natural Protegida (ANP; 10\%). También dieron como opciones de conservación que hubiera constantes pláticas de educación ambiental, que se regule la práctica de la cacería, que haya vigilantes ambientales bien capacitados y que la protección animal se haga presente en el pueblo. Todas estas respuestas dando un total de $3 \%$ cada una; igual se mencionó la prohibición de la cacería, que hubiera una asociación de protección ambiental y que se establezca una veda estricta en todas las especies de animales dentro del ejido.

Como resultado de lo mencionado, un $30 \%$ de la muestra estaría dispuesto a participar de manera voluntaria en cualquier proyecto de conservación que se establezca en la comunidad, un $27 \%$ participaría con remuneración económica y otro $27 \%$ apoyaría asistiendo a pláticas y talleres ambientales; un $13 \%$ participaría de todas las maneras posibles y un $3 \%$ no participaría en nada.

Las entrevistas que se aplicaron fueron consistentes con la información recabada en las encuestas. A pesar de ello, los entrevistados creen que falta bastante información sobre conocimiento de servicios ambientales, de conservación, de manejo ecológico y agroforestal, además de que hace falta añadir un valor cultural y consiente sobre el cuidado del medio ambiente, promover el ecoturismo y la conservación de bosques con cursos, pláticas y ayuda económica.

\section{Discusión}

\section{Inventario de mastofauna}

Las 10 especies de mamíferos registradas representa el $1.83 \%$ de las especies reportados en México y $7 \%$ documentadas en el estado de Nayarit. El esfuerzo de muestreo de 1,260 días trampa se consideró adecuado, debido a que otros trabajos se obtuvieron resultados similares, como el de Medina-Torres (2015) en Chihuahua, quien registró 11 especies en 2,027 días trampa y Pérez-Irineo y 
Santos-Moreno (2012) quienes reportaron 15 especies con 1,156 días trampa en una localidad de Oaxaca. La cantidad de especies encontradas puede variar por la temporada, disponibilidad de recursos para las especies y las zonas muestreadas ya que en los trabajos mencionados las cámaras-trampas fueron colocadas en distintos tipos de vegetación. A diferencia de este estudio en el que se muestreó de manera homogénea en el cerro El molote, lo que podría justificar la ausencia de algunas especies que si fueron mencionadas en las encuestas aplicadas por la comunidad.

Algunas de las especies como el mapache $(P$. lotor) son comúnmente encontradas en lugares con disponibilidad de agua, son más abundantes en áreas boscosas y obtiene la mayoría de su alimento en lugares asociados a cuerpos de agua (Escobar-Anleu, 2015; Lotze y Anderson, 1979). El cerro "El molote", a pesar de ser una zona boscosa y húmeda, no tiene ningún cuerpo de agua, es posible que sea éste el motivo por el cual no se pudiera documentar. Al platicar con las personas de la comunidad, mencionan que la presencia de mapaches ocurre en una zona más baja por donde están algunos ríos. Para el caso de los cánidos, las personas encuestadas argumentaron que los avistamientos de coyote y la zorra gris se dan principalmente a pie de carretera, lo cual coincide con Bekoff (1977), quien postula que el coyote suele preferir zonas despejadas como campo abierto y prados. La zorra gris es un caso parecido al del coyote, es poco común en bosque, prefiere los bordes de bosque y tierras de cultivo (Escobar-Anleu, 2015).

La presencia del zorrillo no fue documentada por las cámaras-trampa, aunque las personas de la comunidad si lo reportaron en la encuesta. Es probable que, hizo falta extender el muestreo más tiempo o hacia otros ambientes, ya que de acuerdo con Escobar-Anleu (2015), esta especie es más común en los bordes de bosque, cañones rocosos, pantanos, pastizales, maleza y hábitat ripario. Asimismo, para el caso del lince (Lynx rufus) Larivière y Walton (1997) argumentan que se puede encontrar en distintos ambientes, desde zonas áridas hasta bosques templados, sin embargo, su presencia está estrechamente relacionada con la abundancia de presas, principalmente de lagomorfos, grupo que tampoco fue registrado por las cámara-trampa en la zona muestreada.

Por otra parte, el tlacuachín tampoco fue captado por las cámaras pues las personas encuestadas definieron que $T$. canescens se observan frecuentemente entre las ramas de las plantas de café. Se han tenido registros en bosques caducifolios, matorrales, praderas de tipo sabana y en bosques secundarios, también se encuentra en las tierras de cultivo y huertos, incluyendo los campos de caña de azúcar, plátanos, papaya, mango, entre otros (Hernández-Cardona et al., 2007; Zarza et al., 2003).

\section{Abundancia}

La abundancia de especies registrada es similar a la documentada por otros autores en cafetales, selvas y bosques mesófilos. Por ejemplo, Aranda et al. (2012) mencionan que las especies con mayor porcentaje de abundancia en una zona de bosque mesófilo de Jalisco-Colima son el coatí ( $N$. narica), el venado cola blanca (O. virginianus), y el pecarí de collar ( $D$. angulatus), así como la presencia de grandes depredadores como el $P$. concolor, $P$. onca, $L$. pardalis y $H$. yagouaroundi. Mientras que Hernández-SaintMartín et al. (2013), postulan que el venado cola blanca y el coatí son presas potenciales para el jaguar y el puma; es de esperar que la abundancia relativa de herbívoros sea mayor a la de los grandes carnívoros, puesto que los grandes carnívoros como los felinos, son mamíferos que naturalmente muestran densidades bajas (Botello et al., 2008). Las especies que presentaron menores frecuencias mencionadas por Aranda et al. (2012) son el armadillo nueve bandas ( $D$. novemcintus) y el jaguarundi, lo cual coincide con el presente trabajo, ya que $H$. yagouaroundi tuvo un solo registro a lo largo de nueve meses de muestreo. En el caso de $D$. novemcintus, el uso de cámaras-trampa se considera un mal método para el muestreo de esta especie, a pesar de eso se obtuvieron registros incluso con el mismo porcentaje de abundancia que pecarí de collar $(D$. angulatus) y el puma $(P$. concolor; ver Figura 3). Lira-Torres y Briones-Salas 2012, registraron al armadillo nueve bandas $(D$. novemcintus) y pecarí de collar ( $D$. angulatus) como especies usualmente muy abundantes.

\section{Percepción sobre el uso, la diversidad y con- servación de la mastofauna}

El principal uso que la comunidad le da a los mamíferos es como alimento de acuerdo con el 54\% de respuestas. Las especies cazadas son principalmente el venado, coatí, armadillo, ardilla, jabalí y el mapache, lo cual es consistente con lo observa- 
do en distintos lugares de México y América Latina (Cortés-Gregorio et al., 2013; Medina-Torres, 2015; Parra-Colorado et al., 2014; Racero-Casarrubia y González-Maya, 2014). También se les da un uso medicinal, tal es el caso del coatí, el armadillo, el tlacuache y el zorrillo, lo cual coincide con diferentes comunidades de Sinaloa, Chiapas, Oaxaca y Colombia, que usan estas mismas especiesde esta manera. Según Enríquez-Vázquez et al., (2006), Cortés-Gregorio et al. (2013) y Parra-Colorado et al. (2014), el armadillo es usado (la grasa del caparazón) para tratar enfermedades respiratorias como la bronquitis al igual que el zorrillo (la glándula odorífera). En la comunidad el tlacuache es utilizado para quitar salpullido y ampollas. Cortés-Gregorio et al., (2013) reportan que se asocia con el remedio para enfermedades respiratorias y Contreras-Díaz y Pérez-Lustre (2008) con un remedio para prologar la vida. El coatí es utilizado para la cura de cólicos, aunque Enríquez-Vázquez et al. (2006) reportan que se asocia a la cura para otros males, como por ejemplo la impotencia sexual masculina y la debilidad sexual. El tráfico ilegal de especies también fue parte de las respuestas, donde el armadillo es la principal especie para la venta por sus usos medicinales y en algunos casos como animal de ornato.

Gracias a declaraciones de algunos miembros de la comunidad, fue posible determinar que quienes clasificaron al jaguar como peligro para la biodiversidad o que no existen amenazas para la fauna, son personas que se dedican a la cacería. Más de la mitad de los encuestados clasificó la cacería ilegal como el mayor problema para la biodiversidad, actividad que se ve favorecida dados los usos y costumbres de los pobladores, que hacen uso de la fauna silvestre, aunado a la cacería deportiva.

En distintas partes del país y de América Latina, existe el conflicto Jaguar-humano. La interacción del ser humano con mamíferos carnívoros, ha sido clasificada como un conflicto, ya que estas especies son perseguidas al atribuirles un daño a los animales domésticos o simplemente por temor de ser atacados por los jaguares. Esta problemática es resultado de la perturbación humana y por la ausencia de presas naturales (Parra-Colorado et al., 2014). Al analizar los resultados de este estudio, se detecta una problemática dentro de la comunidad, ya que hay un porcentaje mayor de la población que identifica al jaguar como un depredador sobre los animales buenos que los perros ferales. En México las poblaciones de perros ferales se han convertido en un problema, ya que para sobrevivir se han con- vertido en buenos cazadores de animales silvestres de talla pequeña, mediana y hasta de grande (Weber, 2010).

Con los datos obtenidos por medio de las encuestas y entrevistas. Se puede deducir que la percepción sobre los mamíferos dentro de la comunidad es buena, se identifican 20 especies de mamíferos por su nombre común, hay antecedentes de uso de estas y la mayoría de los encuestados consideran la conservación como un aspecto muy importante. Además, ven a los mamíferos como una oportunidad viable para generar derrama económica; sin embargo, también se puede percibir una opinión dividida, pues prevalece un pequeño sector de la comunidad que practica la cacería más allá del autoconsumo y quienes no muestran ningún tipo de interés por la conservación de la naturaleza.

El trabajo con las comunidades suele ser complicado por muchas cuestiones, ya que no toda la comunidad está dispuesta a participar de manera activa. Los conocimientos que poseen los habitantes son valiosos; la mastofauna aún forma parte de su entorno diario, por lo que en este aspecto radica la importancia de la participación de las comunidades en los inventarios biológicos. El trabajo académico se ve enriquecido y la comunidad realiza un ejercicio de reflexión sobre los beneficios ambientales, económicos y culturales que conlleva el conocimiento de la biodiversidad.

\section{Conclusiones}

Los habitantes de la comunidad de Cumbres de Huicicila tienen un buen conocimiento sobre la mastofauna que se distribuye en la región, ya que reconocen las diez especies registradas mediante el video-trampeo y además reconocen diez especies más de mamíferos que no pudieron ser captadas por las cámaras-trampa. La comunidad resguarda valiosos conocimientos tradicionales sobre los mamíferos, principalmente los de uso alimenticio y medicinales, enseñanzas aprendidas de manera empírica mediante la observación y replicación de los conocimientos tradicionales; esto es importante ya que puede influir a que la mayoría de la población esté dispuesta a que se ejecuten programas de conservación para las especies nativas. El jaguar $(P$. onca) resultó ser una especie con la que se tiene problemas dentro de la comunidad, ya que se identificó un evidente conflicto humano-jaguar entre algunas personas que lo consideran un peligro ha- 
cia su persona y las especies de fauna silvestre que ellos denominan buenas, al formar parte de la dieta del jaguar. Es importante señalar que aún hay personas, a pesar ser una minoría, que consideran al jaguar como un animal malo. Asimismo, existe una fuerte problemática de cacería ilegal, relacionada con ese pequeño grupo de personas. Por lo cual, es importante fomentar la investigación y la educación ambiental en esta zona con el fin de mostrar los beneficios que pueden traer para la comunidad, la conservación de la biodiversidad y la presencia de depredadores tope en los ecosistemas.

\section{Agradecimientos}

Agradecemos a las autoridades y los pobladores de la comunidad de Cumbres de Huicicila por su amabilidad y las facilidades que se otorgaron durante la investigación. Al señor Antonio Cuevas Salazar, por su apoyo, participación y gran entusiasmo en esta investigación, sin ello, esto no hubiera sido posible. Al señor Rodrigo Becerra Medrano, por su ayuda, disposición al permitir que este proyecto se desarrollara dentro de sus predios privados. Al señor Gustavo de Luna, por su siempre valiosa participación, apoyo y sincera amistad. A las familias de Don Jorge Talamantes, Francisco Mercado, además de las familias Cuevas Salazar, Cuevas Rivera, Cuevas Velázquez y De Luna López que siempre tuvieron las puertas abiertas de sus casas, por la amabilidad, la confianza y el apoyo que nos brindaron.

\section{LITERATURA CITADA}

Alcalá, S.A.A., y G. De la Riva Hernández. 2016. Uso tradicional de fauna silvestre en las serranías del occidente del Estado Aguascalientes, México. Etnobiología, 14:20-36.

Aranda, M., Botello, F. y L. López-de Buen. 2012. Diversidad y datos reproductivos de mamíferos medianos y grandes en el bosque mesófilo de montaña de la Reserva de la Biosfera Sierra de Manantlán, Jalisco-Colima, México. Revista mexicana de biodiversidad, 83:778-784.

Bautista-Hernández, C.E., S. Monks y G. Pulido-Flores. 2013. Los parásitos y el estudio de su biodiversidad: un enfoque sobre los estimadores de la riqueza de especies. Estudios científicos en el estado de Hidalgo y zonas aledañas. Zea Books, Lincoln, Nebraska, 13-17.
Bekoff, M. 1977. Canis latrans. Mammalian species, 79:1-9.

Bocco G., A. Velázquez y A. Torres. 2000. Ciencia, Comunidades Indígenas y Manejo de Recursos Naturales. Un caso de Investigación Participativa en México. Interciencia, 25:64-70.

Botello, F., V. Sánchez-Cordero y G. González. 2008. Diversidad de carnívoros en Santa Catarina Ixtepeji, Sierra Madre de Oaxaca, México. Avances en el estudio de los mamíferos de México, 2:335-354.

Carmona, G. 2015. Estudio de prevalencia para la determinación de correlación entre edad y enfermedades físicas y construcción del modelo de regresión múltiple por Stepwise en el cuerpo policial de Costa Rica. Tesis. Universidad Autónoma de Barcelona. Barcelona, España.

Ceballos, G. y G. Oliva. 2005. Los mamíferos silvestres de México. Fondo de cultura económica, Comisión Nacional para el Conocimiento y Uso de la Biodiversidad. México, D.F.

Ceballos, G., y J. Arroyo-Cabrales. 2012. Lista actualizada de los mamíferos de México 2012. Revista Mexicana de mastozoología nueva época, 2(1):27-80.

Chávez, C., A. De la Torre, H. Bárcenas, R.A. Medellín, H. Zarza y G. Ceballos. 2013. Manual de fototrampeo para estudio de fauna silvestre. El jaguar en México como estudio de caso. Alianza WWF-TELCEL-UNAM. México.

Colwell, R.K. 2013. Estimates, version v. 9.1.0: statistical estimation of species richness and shared species from samples, sotware and user's guide. Mansield, Connecticut: University of Connecticut, Mansield.

CONABIO, 1998. La diversidad biológica de México: Estudio de país, 1998. Comisión para el Conocimiento y uso de la Biodiversidad. México.

Contreras-Díaz, R.G. y M. Pérez-Lustre, M. 2008. Etnoecología de mamíferos silvestres y los zapotecos del municipio de Santiago Camotlán, Villa Alta, Oaxaca. Etnobiología, 6:56-67.

Cortés-Gregorio, I., E. Pascual-Ramos, S.M. Medina-Torres, E.A. Sandoval-Forero, E. Lara-Ponce, 
H.H. Piña-Ruíz, y G.E. Rojo-Martínez. 2013. Etnozoología del pueblo Mayo-Yoreme en el norte de Sinaloa: uso de vertebrados silvestres. Agricultura, sociedad y desarrollo, 10:335-358.

Enríquez-Vázquez, P., R. Mariaca-Méndez, Ó.G. Retana-Guiascón, y E.J. Naranjo-Piñera. 2006. Uso medicinal de la fauna silvestre en los Altos de Chiapas, México. Interciencia, 31:91-94.

Escobar-Anleu, B. 2015. Riqueza de mamíferos medianos y mayores en cafetales y bosques de tres reservas naturales privadas (San Jerónimo Miramar-quixayá, pampojilá-peña flor y Santo Tomás Pachuj) de la Reserva de usos múltiples de la cuenca del lago de Atitlán. (Tesis de Licenciatura) Universidad de San Carlos de Guatemala, Facultad de Ciencias Químicas y Farmacia.

Godínez, O. 2014. Patrones de Actividad Espacio-Temporal de los Ungulados de la Reserva de la Biosfera El Triunfo, Chiapas, México. Tesis de Licenciatura. Universidad Michoacana de San Nicolás de Hidalgo. Morelia, Michoacán.

Hernández-Cadena, F. 2015. Diversidad de mamíferos del estado de Nayarit. Tesis de licenciatura. Universidad Autónoma De Nayarit. Nayarit, México.

Hernández-Cardona, A., L. A. Lago-Torres, L, Ibarra-González, J. C, Faller-Menéndez y Y.Y. Pereyra-Arellano. 2007. Registro del tlacuachín (Tlacuatzin canescens) en el área de conservación El Zapotal, en el noreste del estado de Yucatán. Revista Mexicana de Mastozoología, 11:85-90.

Hernández-SaintMartín, A.D., O.C., Rosas-Rosas, J., Palacio-Núñez, L.A., Tarango-Arámbula, F., Clemente-Sánchez y A.L. Hoogesteijn. 2013. Activity patterns of jaguar, puma and their potential prey in San Luis Potosi, Mexico. Acta Zoológica Mexicana (nueva serie), 29:520-533.

INEGI, 2010. Censo de población y vivienda, 2010. http://www.inegi.org.mx/sistemas/consulta_resultados/ iter2010.aspx?c=27329\&s=est.

Larivière, S., y L. R. Walton. 1997. Lynx rufus. Mammalian species, 563:1-8.

Lira-Torres I. y M. Briones-Salas. 2012. Abundancia relativa y patrones de actividad de los mamíferos de los chimalapas, Oaxaca, México. Acta zoológica mexicana (nueva serie), 28:566-585.
Lotze, J.H., y S. Anderson. 1979. Procyon lotor. Mammalian species, 119:1-8.

Lozano-Rodríguez, L.A. 2010. Abundancia relativa y distribución de mamíferos medianos y grandes en dos coberturas vegetales en el santuario de fauna y flora Otún Quimbaya mediante el uso de cámaras trampa. Tesis de Licenciatura. Pontificia Universidad Javeriana, facultad de Ciencias.

Medina-Torres, S.M., E.L. Gastélum-Vizcarra, E. Lara-Ponce y H.H. Piña-Ruiz. 2015. Inventario participativo de mamíferos silvestres en el ejido San Ignacio, municipio de Morelos, Chihuahua. Acta Zoológica Mexicana, 31:221-233.

Medina-Torres, S.M., Cortés-Gregorio, I., Lara-Ponce, E., y E.A. Sandoval-Forero. 2016. Uso de mamíferos silvestres entre yoremes y yoris de $\mathrm{El}$ Fuerte, Sinaloa, México. Agricultura, sociedad y desarrollo, 13:529-545.

Moreno, C.E. 2001. Métodos para medir la biodiversidad. M\&T-Manuales y Tesis SEA, Zaragoza, Vol.1. 84(922495), 2.

Myska, P. 2015. Guía de campo de Anfibios, Reptiles, Aves y Mamíferos de México Occidental. Viva Natura, México.

Parra-Colorado, J.W., Á. Botero-Botero y C.A. Saavedra-Rodríguez. 2014. Percepción y uso de mamíferos silvestres por comunidades campesinas andinas de Génova, Quindío, Colombia. Boletín Científico. Centro de Museos. Museo de Historia Natural, 18:78-93.

Pérez-Irineo, G. y A. Santos-Moreno. 2012. Diversidad de mamíferos terrestres de talla grande y media de una selva subcaducifolia del noreste de Oaxaca, México. Revista Mexicana de Biodiversidad, 83:164-169.

PHINA (Padrón e Historial de Núcleos Agrarios). 2017. Recuperado de http://www.ran.gob.mx/ran/index.php/sistemas-de-consulta/phina.

Racero-Casarrubia, J., y J. F. González-Maya. 2014. Inventario preliminar y uso de mamíferos silvestres por comunidades campesinas del sector oriental del cerro Murrucucú, municipio de Tierralta, Córdoba, Colombia. Mammalogy Notes, $1: 25-28$. 
Ramírez-Pulido, J., N. González-Ruiz, A.L. Gardner y J. Arroyo-Cabrales. 2014. List of recent land mammals from Mexico, 2014. Especial publications, Museum of Texas Tech University, 63:1-69.

Ramírez-Silva J.P., D, Hernández De La Rosa, F.J. Hernández-Cadena y G. Woolrich-Piña. 2016. Conservación de los mamíferos de Nayarit. Pp. 311-328, en: Riqueza y conservación de los Mamíferos en México a nivel estatal (Briones-Salas, M., Y. Hortelano-Moncada, G. Magaña-Cota, G. Sánchez-Rojas y J.E. Sosa-Escalante, eds). Instituto de Biología, Universidad Nacional Autónoma de México, Asociación Nacional de Mastozoología A.C. y Universidad de Guanajuato, Ciudad de México, México.

Rosas-Rosas, O.C., H.S. Martin, J. I, Olvera-Hernández, J. D. Guerrero-Rodríguez, E. Aceves-Ruíz y L. A. Tarango-Arámbula. 2015. Monitores comunitarios para la conservación e investigación participativa en áreas naturales protegidas. Agroproductividad, 8:56-61.

Semarnat (Secretaría de Medio Ambiente y Recursos Naturales). 2010. Norma Oficial Mexicana NOM-059-ECOL-2010, Protección ambiental-especies nativas de México de flora y fauna silvestres-categorías de riesgo y especificaciones para su inclusión, exclusión o cambio-lista de especies en riesgo. Diario Oficial de la Federación. México, 30 de diciembre, del 2010:1-77.

Weber, M. 2010. Perros (Canis lupus familiaris) y gatos (Felis catus) ferales en la Reserva de la Biosfera Los Petenes, Campeche, México: Diagnóstico, efectos en la fauna nativa y perspectivas de control. Informe final proyecto SDP-18-2008 PNUD-CONANP-ECOSUR. Campeche, México.

Zarza, H., Ceballos, G. y M.A. Steele. 2003. Marmosa canescens. Mammalian Species, 1-4. 